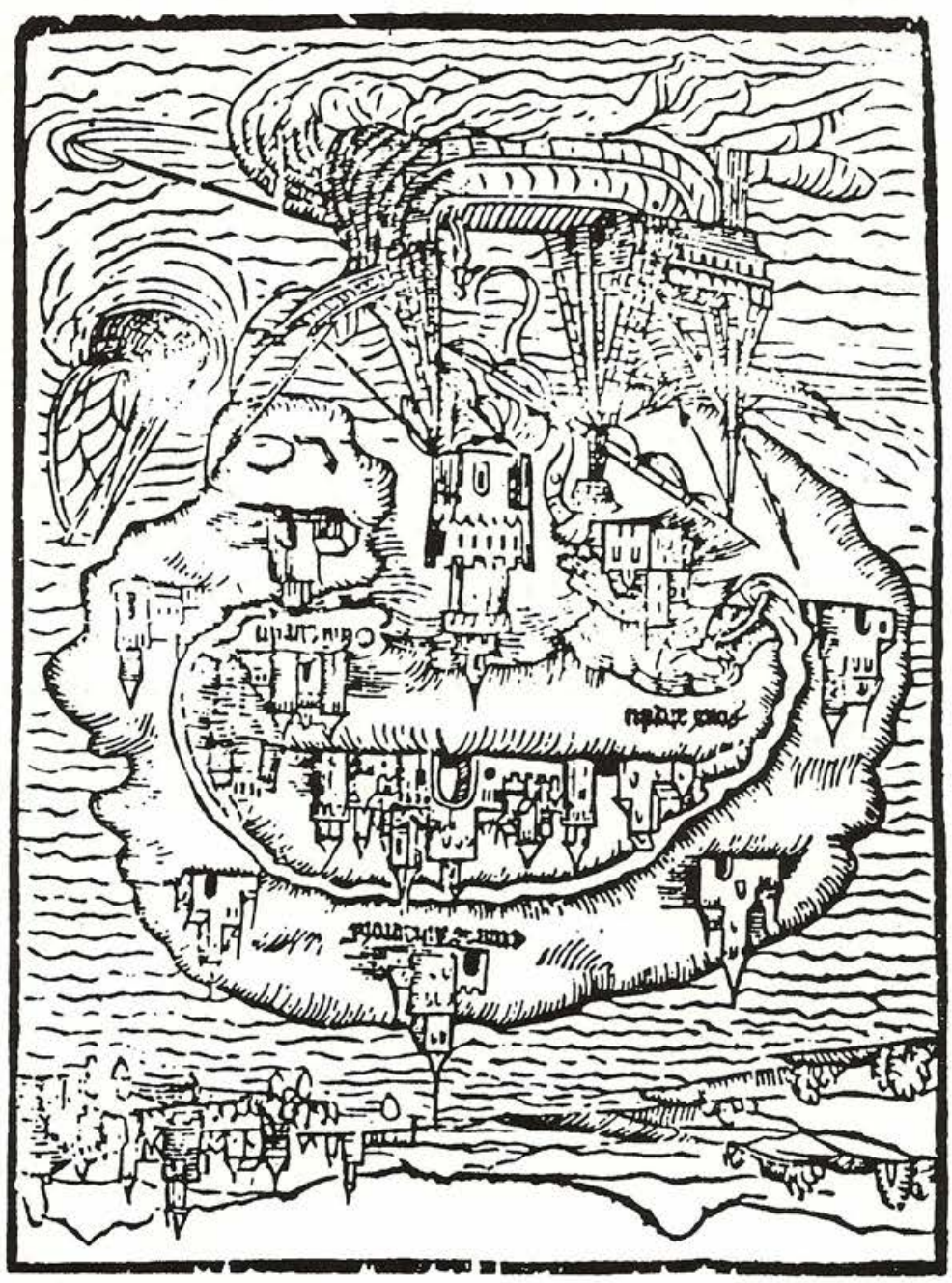




\section{Two Visions of Utopia}

\section{David J Fausett}

The 'two visions of utopia' that I wish to discuss here are critical ones: interpretations of utopian theory, rather than any particular idealisms or imaginary worlds. They refer to two distinct periods in literary and social history, the pre- and post-Enlightenment; and to the two distinct types of utopian writing produced by those periods. Because of the utopia's close association in any period with historical and ideological realities, an understanding of the shift in attitudes and styles that occurred in the Enlightenment is essential in approaching the question as to why we should now be in a third phase in relation to utopianism, in which no such form of idealism is any longer taken seriously, either as positive idealism in the literal sense, or as negative idealism in the satirical sense. Something that utopian writing stood for throughout its history, in both of these modes, seems to have slipped away from the modern consciousness, and that is really what is posed as a question within the continuing debate over utopian theory.

Utopianism itself has in all its forms come to be regarded as a sort of 'closet totalitarianism': but it should not be supposed that the theory or interpretation of it has similarly become a dead issue: far from it. The issue is just as immediately relevant - particularly in situations of post-colonial ethnic integration such as New Zealand faces - as it was for Plato. Indeed, the 'question of utopia' in this abstract, critical sense is the single most significant point of reference in the problematics of the so-called 'closed' and 'open' societies.

So to begin with, it must be admitted that utopian literature is no longer much in vogue, except as an object of analysis in literary criticism and social theory. Some landmarks of the genre are generally known, such as Plato's Atlantis, More's Utopia, Campanella's City of the Sun, Bacon's New Atlantis, Swift's Gulliver, the 19th-century idealisms of Saint-Simon and Fourier, and the 20th-century antiutopias of Wells and Orwell. But these are only the tip of a vast iceberg of productions, mainly in French and English literature; and they do not at all represent a single genre. There are two distinct categories of works commonly lumped together as utopias, and an adequate approach to this form of writing must from Thomas Moore, Utopia, Frontis piece 
making the distinction between the 'classical' genre of pre-Enlightenment times, and the more familiar modern utopia.

This distinction is necessary because utopian writing is closely related to ideology and theories of social structure, and because these, as its referents, have undergone a major change during the Enlightenment, of which the two visions are symptomatic and which, therefore, can be analytically approached through the study of utopianism.

The irony in this distinction is that the uncritical assumptions that subsist because utopianism is now a relatively minor field of literature, tend to assimilate all utopianism to the modern variety; whereas in fact it is only the classical variety that should strictly be called a utopia at all, for reasons that will hopefully become clear as we examine what was at stake in the two forms of utopianism.

So, there have been two phases of utopian writing, and the passage from one to the other can contribute to our understanding of the Enlightenment and of the 'crisis' of the advent of modernity. But in this enterprise we are faced at the outset with a major problem of definition: what, finally, is a utopia, in the strict sense? The uncritical assumptions just referred to equate utopianism in general with the modern anticipatory form of idealism: whether inspired, critical, fanatical, constructive or despairing, it is in all cases regarded as a vision of some sort of world to come. It is a future-oriented idealism. But this is not the strict and original sense of the genre; it is a travesty of it, taking over the classical utopia's place when the latter became extinct in the course of the Enlightenment. The original sense was that conveyed by the Greek root of the word, ou-topia, meaning a 'no-place; which was the name invented by Thomas More to describe a society locatable in discourse, but unlocateable in reality, as a physical 'point' on the earth's surface. This rhetorical gesture had arisen alongside the problematical progress of geographical discovery, as an expression of the corresponding problematic of local territorial space and, implicitly, of the relation between territorial configuration and social structure. It was this significant relation that became invisible or irrelevant when geographical knowledge ceased to be a problem, so that the literary genre that it had supported fell into disuse.

Effectively, the utopia arose as an expression of the way in which the architectonics of social structure is patterned on that of physical space, or territory; and by pursuing this relation through its Enlightenment transmutation to found a significantly modified genre, we can gain an insight into the nature of the changed social structure that was engendered by the new, global configuration of territorial space that was inaugurated at that time, by the virtual completion of the discoveryprocess. 
In its eschatological bearing, the modern utopia has at the same time taken over the function of another major casualty of the Enlightenment, religious ideology; while the new form's profound collusion with the statist programmes culminating notably in the American and French constitutions has not escaped theorists such as Marx. Indeed, it is just this disturbing proximity of modern utopianism to modern ideological orthodoxy that in large part determines the seemingly 'hysterical' refusal by the philosophical Establishment to reckon seriously with utopianism. And yet we are dealing with the crucial point of articulation between the architectonics of the physical and social realms, as noted and as is suggested by the double use of this word, in architecture and in philosophy.

Such refusal of utopianism may well be justified in the case of the 'naive' idealism of the modern utopia: and one would readily concede that it is less a matter of (re-)creating anything in the realm of social structures, than of understanding empirical processes themselves; or, at least, that the latter understanding is the necessary prerequisite to any effective social action, and is a task that would, if taken to its limit, inhibit all such action. But that understanding, and that essentially passive relation to empirical sociological reality, was precisely what the classical utopia represented, by contrast with its modern avatar. By re-programming our reading of the classical genre in accordance with what we are able to reconstruct of the general pre-Enlightenment 'vision of the world', we can begin to theorise what has been at stake in the turn to sociological and philosophical modernity, and to realise the implications; of the profound collusion of modernity with the anticipatory or activist form of utopianism.

For the modern world is itself, quite frankly, a utopia; or more precisely, a pantopia: a societal form determined by global inclusiveness. By contrast, the classical world and world-view (and their articulation together as classical utopia) was determined by the residually primitive closedness of the idealising reference back to local, partial culture as the source of models of behaviour, of meaning and of authority in general. The classical utopia, accordingly, was less concerned with the representation of new futures than with that of the general process of 'closed' reference to the cultural past. The Enlightenment, or crisis of modernity, was the profound shift from the one concept of social existence to the other: an integral disinvestment of the primitive valourisation of the past, and a turning to the valourisation of unknown, conjectural futures. In the same movement occurred the definitive turn from collectivism to individualism, and from socialism to materialism; which can be seen, by analysing the concept of metaphysics, to be fundamentally irreconcilable sets of goals.

Some explanation is perhaps indicated, of the use here of the terms 'primitive', 'classical', and 'modern'. 'Primitive' refers, not to any judgement of developmental value, but to the underlying etymological sense of the word, as it is still used in 
French for example: of that which is first or original. 'Classical' refers, not to Mediterranean Antiquity itself (although the phenomena in question had their beginnings there), but to the entire history of emancipation from the 'primitive' both in society and in utopian writing, down to the definitive rupture with it in the Enlightenment. 'Modern' in the present usage refers particularly to the latter institution of future-oriented world-view, rather than to the whole of the postRenaissance period, for example: the original 'primitivist' or collectivist bias having survived in effect down to the Enlightenment and the sea-change instituting the world-view we now own. In utopianism as in its societal referent, then, 'classical' and 'primitive' here signify a general mode of fidelity to received patterns; and for this reason I use the umbrella term 'classico-primitive' to cover this meaning. 'Modern', on the other hand, signifies the mental horizon determined by the loss or irrelevance of such societal patterns and, secondarily, of the literary means of representing them.

Another clarification is perhaps called for, of the assumption just made about the utopia's 'societal referent'. Utopian writing is generally recognised as being a form of ideological predication; and yet the distinction between its aims and styles in the two periods of its production is less well-known. Accordingly, that to which such writing refers is assumed uncritically to have always been the same. What attention to the distinction between classical and modern utopianism can help to illuminate is the profound shift in collective goals that the Enlightenment represents, and the reason why it has generated social crisis. Indeed, the very fact that it has generated crisis can only be verified, from within the modern ethos, by reference to the latter's classico-primitive exterior; since it is in the nature of all ideology to imply that the given is the 'best of all possible worlds', others being merely imperfect realisations of it.

The social referent, then, was a goal of a different order before the Enlightenment, to what it has become since: it was at that time a primary goal, by contrast with the relative (and progressive) primacy in modern times, of material-economic goals. It is this distinction that can be made by analysing the difference between the two orders of utopianism, and that lies at the heart of the question as to why the advent of such a modernity should have been a 'crisis'. Reference to social history helps to define the goals that have historically been pursued by man. It is commonly supposed, for example, that because technology and material-economic concerns in general are the primary concern of modern man, that it was always so: that 'such is the nature of man' (Sahlins and others not withstanding). The scientific pursuit of the origins and nature of humanity is dominated by this base-assumption of a primary technical orientation: hence 'homo faber', 'homo economicus', etc., and the whole paleontological orientation (which, admittedly, is also in large part shaped by the nature of the surviving evidence). And yet one need only look sympathetically at the evidence of ethnology and of ethology, to realise that the logically prior 
goals, where any social organisation is concerned, are to do with the maintenance of that organisation itself: with the technics of social structure.

Viewed in these pre-modern terms, the 18th-century crisis can be seen to resemble in the first instance a social, rather than an industrial, revolution. It inaugurated an industrial revolution, but that was not in itself the primary cause. The same tautology or blinkering-effect dominates the perception of human history in the context of its 'origins' themselves: as a retrojection of the effects generated by historical events, in the assumption that these effects were teleologically the cause of the events, forcing them to occur by their own inevitability. Such modes of thought are determined by the thinker's encapsulation within his/her own historical mentality: a kind of inbuilt limitation to any thought about a historical or epistemological other that links it more or less closely to myth.

The classical utopia was relatively 'up front' about this inherently mythical bearing of ideology; as already noted, an explicit recognition of it was built in to its rhetorical mode, in the form of the metaphor based on geographical knowledge. From the Greek beginnings of what in modern times has become social science, speculation about social or cultural otherness was prefaced in this way by the satirical gesture of a play on social ideality, on its 'unlocateability' in the real world. As a complement to serious ideological or primitivist speculation, the utopia implicitly posed the prior question, what could we know about it? Who are 'they', or 'we'? Where are the boundaries between cultural sameness and otherness? Are categories such as 'man' and 'society' universals?

These questions bear, as mentioned, on the relative interpretations of classical and modern utopianism: the latter is concerned with anticipatory speculation (whether it be good or ill that is anticipated), and is linked to the general 'planifying' approach to social as well as to material-economic processes, that is progressively realised as the 'essence of the modern'. From within such a mentality it is inevitable that the classical utopia will tend to be perceived in the same light.

And yet it is the classical utopia itself that points to the logical flaw in that uncritical perception, by means of its discursive essence as a representation of (an imaginary) society. As such it abstracts and depicts the social technicity which was originally the primary one: that of which the dissolution causes the materialist primacy we now know to be precipitated into primacy. The logical relative primacies of these factors can be seen by projecting the 'chicken-egg' aporia back to the hypothetical origins, where there was virtually no material-economic technicity at all; but very definitely a complex technicity of social organisation, as modern ethology well knows. The classical utopia can thus be employed to deconstruct its own uncritical reading by a post-classical age and, in the process and in view of its demise with the advent of modernity, it can serve as the crucial symptom of the great 'articulation' 
in social history, between these two worlds and world-views. In a word, there are two 'visions of utopia', because there have been two fundamentally different societal modes.

Hence the magnitude of the shift in structural patterns that the 18th century crisis represents. To put it in Popperian terms, it is the turn from the closed society to the so-called 'open society'. The classical utopia was, more than anything else, a figuring or iconic representation of the closed society: which is to say, of the closedness of the human or religious system, whereby it generates and sustains the specifically human form of semanticity, or language. The latter's distinctive quality is that it is arbitrary: a product of convention, rather than of nature. Linguists have analysed at length the phonetic and grammatical aspects of man's 'articulated language', without generally realising that the articulation in question is first and foremost that of ethical topoi, of relations of individuals and collectivities.

In semiotic processes, meaning is generated and exchanged within a 'closed circuit' of communication, analogous to that of the words' familiar technical usage; and this closedness is what generates the effect of meaning, which could be called 'sponsibility' or sponsion in order to emphasise that it arises out of, and refers back to, ethological configurations. It is because of this dependence for semantic ratification on a 'closed circuit, that there can be no serious question of an 'open society': since that would be an absence of society or meaning, in the human sense; a relapse into animal sociality. The question is a more complex one, involving the definition of relative degrees of closedness and openness; and involving therefore analysis of the historical interplay of these factors, as the result on the one hand of socio-cultural conservatism, and on the other of the forces of culture-contact and assimilation.

As mentioned, the classical utopia's role in representing this interplay, expressed as its spatial metaphor, was central to the pre-Enlightenment understanding of the social process. The ideal republic was effectively a 'pre-contact' society - ie. one miraculously preserved from inclusion within the Western monoculturalist orbit; and it was in this that it was able represent that 'primitive' or essentially human quality that the classical mentality residually valourised: the inherently closed and arbitrary nature of human ideology. There are two main ramifications of this interpretation: firstly, the fact that utopian writing arose as a commentary on the process of cultural assimilation, geographical exploration and commercial exploitation known as the Western expansion; and secondly, that the socio-cultural relativity or problematicity which inspired that commentary has, since the Enlightenment, virtually disappeared from Western man's consciousness; a disappearance inseparable from that of the classical utopia itself.

These two aspects of the question, the geographical and the socio-structural, are 
mutually significant in a way that tends, furthermore, to have slipped below the modern horizon of readability, within which there can be no social ideal (or society at all) except the future universal one, needing to be 'made'. That significance or double architectonics of spatial and social configuration in fact holds the key to the question as to why the momentous change from social passivism to activism should have occurred at all. If the advent of Western monoculture is a process of restructuring on a progressively larger scale, leading to a uniform global or 'open' society, why should the latter not simply be a fully-realised version of what each of its constituent social elements was striving towards? Such is, indeed, what the prevailing ideology would have us believe. But it is not the case, because the structural conditions are decisively different in the two cases. As the assimilation process intensifies and residual classico-primitive effects are diluted, a new form of society emerges, based on the principle of 'general equivalence'; and it becomes clear that in this process two and two do not add up to four, since the 'twoness' of the twos had been a structural effect of their mutual relationship. This is why monoculture cannot be 'human' society in the traditional sense of the word.

The difference, as noted, has to do with the inherently 'closed' nature of the human ideological formation, which depends on a situation of cultural relativity: of the mutual difference that provided the structuring force. Human ideology or semanticity was originally structured as such, as the formal exclusion of a competing social exterior; a process theoretically inseparable from the role of territory in generating and supporting social 'insularity'. The classical utopia was the pre-Enlightenment expression of that relation of analogy.

What happens, though, if the perspective of local difference is put out of play, by the progress of territorial assimilation, to the point where the entire world is effectively 'known', or included in a 'global village'? Then, an equal and opposite reaction sets in, and an involution occurs in the socio-structural process that had been primarily based on territorial exclusion. This is the perspective that was produced by the 18th-century closure of the global circle, as a 'crisis of consciousness' and which, since that time, has progressively hardened into a crisis of social disintegration.

Such effects are the necessary corollary of a virtual realisation of the monoculturalist process determined in the first instance by the loss of the physical basis of cultural difference: spatial isolation. If the residual 'closedness' or relativity we still know were to melt down completely into a uniform world culture, not only would all the problems of cultural integration be solved, but in the same movement humanity itself would disappear, in the form that we know it. And yet, that is clearly what is happening; the trend towards 'post-structural' uniformity appears inevitable. The specifically human effect, generated initially as a passage 'beyond the physis' of territory and into the metaphysics of religious ideology, subsists 
within the 'society of representation' (or political, monoculturalist society) only as, precisely, an effect of representation. It is not spontaneously regenerated there, because the structural preconditions are absent. Hence, the 'crisis'.

What, then, can the classical utopia tell us about where this process leads to? Firstly, there is the historical demise of the genre itself, which, as mentioned, is highly significant. Secondly, there are individual works which, by virtue of their authors' prophetic insight, have satirically portrayed a calculated guess as to the outcome of the process; among these, Gabriel de Foigny's Terre Australe connue is probably the most interesting. Thirdly and most importantly, there is the analytical key that the utopia's play on spatial reality provides, to the enigma of the primary structural process and its relation to territorial configuration. By following through the implications of this analytical perspective, one can begin to see what has been involved in the 'going into reverse' of the primary process.

The intention here is to outline briefly that perspective, noting its implications in terms of a 'lapse' into uniformity, particularly as satirised by the late classical 'austro-hermaphrodite' metaphor, of which the Terre Australe connue just mentioned is most representative. By means of this approach it may be possible to shed some light, not only on the fate of the classical utopia, but also on that of its referent, the classico-primitive form of society; and, by means of this reference 'outside', to approach a theoretical understanding of the modern ethos.

Because there have been two distinct 'visions of utopia' in the abstract sense here intended, hinging on the 18th-century crisis of Enlightenment, it is necessary for the critic or historian of ideas to approach the analysis of utopian theory with a double critical vision, bearing in mind that the pre-Enlightenment world is emphatically not ours. The reason why it is not comes down ultimately to the classico-primitive relation to the organic cultural past, and to the territorial physics that primarily supports such 'local' difference. When the latter was virtually liquidated by the global extension of geographical knowledge around the 18th century, a new form of society defined in terms of an absolute territory was born, or rather realised, as the Western monoculture. The 'open society' or absolutist state was in the first instance rendered possible by this historical opening-up of the primitive closedness of territory; in which the status of the last 'unknown', the Australasian and South Pacific regions and Antarctica, was exemplary. Western geographical and commercial expansion had thus had the sociological effect of reversing the primary process of metaphysical structuration, source of the human phenomenon'.

This is why the modern society predicated as universal, far from being a classicoprimitive 'quasi-utopia', is in fact literally a utopia, or pantopia, in terms of the original usage of the word. The 'closed circuit' that had been figured in its closure 
as the hermeneutics of the 'unknowable' ideal society, and represented metaphorically as a geographical 'non-location', has virtually ceased to exist as such: by virtue, that is, of the removal, of its physical preconditions of insularity or territoriality. A universal society would have no social exterior, just as a global territory has no physical exterior; and, therefore, no structuring force regenerating the metaphysical basis of religious ideology. In other words, there would no longer be the force opposing, and strategically repressing, direct animal sociality. It is the relative lifting of that force, for example, that has conditioned the rise of individualism and of materialism, and of their corollaries such as psychoanalytical theory.

The value of the classical utopia lay in the implicit connection it drew between these sociological effects and their primary basis in geophysical configuration; a perspective which, as noted, has virtually disappeared with the advent of the 'global' overview. Indeed, an involution of the primary social and epistemological forms has occurred: the 'closed circuit' has become definitively opened, tied to no significant place but to all places. The classical genre necessarily disappeared in this movement; its gesture of concrete representation (as imaginary society) of an abstract phenomenon (the human 'closed circuit') being supplanted by the abstract representation (as literary criticism or social science) of a concrete phenomenon: the advent of that real-historical 'utopia', the global monoculture of which we presently inhabit an early phase.

To recall, in conclusion, some of the implications of this process: firstly, the latter is particularly acute at the margins of the monoculturalist vortex, at the interface between what remains of the primary or 'pre-contact' social form, and what has been irrevocably instituted of the secondary form, or 'society of representation' - in other words, in the ex-colonies. Secondly, the base-phenomenon is the reduction to 'general equivalence' which, while initially a material-economic process, becomes beyond a certain limit a socio-structural one, in which the person acquires a status equivalent to that of merchandise, and is propelled towards uniformity and exchangeability in terms of his or her - or more precisely 'its' - social roles. This process affects (secondarily) the metaphysical status of the body and of the relation to the material world. The meaning and fate of the classical utopia provide a clue to such social architectonics, because the latter are determined in all cases by the status of the mediating 'closed circuit'.

Finally, on the subject of architectonics and the significant parallel between the architectural and epistemological senses of the word, let us consider some etymological usages which betray the same base-concept as I have identified in the case of the classical utopia. Take the convent, which has the conventional or arbitrary character of human religious ideology inscribed in its very name, designating a gathering-place in which religion (also implying a quasi-physical 
binding) is practiced; while its cloisters again carry the sense of essential closure, as in the word's kinship to the French 'clôture', which literally means 'closure'. These examples perhaps serve to illustrate the close relation between configurations of physical space and social structure in the classico-primitive conceptuality, that was the basis of classical utopianism: one which in our time is by contrast barely conceivable, so completely has the significance of the primary bounded structure fallen into irrelevance. 